\title{
Perfil clínico e histopatológico do cisto odontogênico calcificante: relato de caso
}

\author{
Hugo Leite Rodrigues Neto* \\ Daniel Amaral Alves Marlière** \\ Marcelo Galindo Silvares*** \\ Luciana Asprino** \\ Rodrigo Alvitos Pereira***
}

\begin{abstract}
RESUMO
O cisto odontogênico calcificante (COC) é considerado como condição patológica benigna de ocorrência rara em maxila e mandíbula, caracterizado por revestimento cístico de células epiteliais odontogênicas, contendo células fantasmas com propensão a regiões de calcificação. Neste artigo, descreveu-se a configuração clínica e histopatológica do COC por meio de relato de caso submetido a tratamento cirúrgico e acompanhamento pós-operatório. Paciente de 11 anos apresentou aumento de volume em região maxilar à esquerda, próxima ao sulco nasolabial e sem sintomatologia dolorosa. Os exames radiográficos indicaram lesão radiolúcida, bem circunscrita e expansiva em maxila, que foi submetida a enucleação associada a ostectomia marginal das paredes corticais ósseas. O histopatológico revelou revestimento cístico com epitélio odontogênico ameloblástico, ninhos espalhados de células fantasmas e regiões eosinofílicas de material compatível com dentinóide. Evidenciou-se, assim, perfil clínico de COC, que pode ser confundido com outras lesões císticas ou tumorais, sendo essencial o diagnóstico por meio de análise histopatológica. A abordagem cirúrgica proporcionou adequado diagnóstico e tratamento. Após acompanhamento clínico e radiográfico, não houve recorrência do COC.
\end{abstract}

Palavras-chave: Cisto Odontogênico Calcificante. Odontogênese. Patologia Bucal. Cirurgia Bucal.

\section{INTRODUÇÃO}

O cisto odontogênico calcificante (COC) é classificado pela Organização Mundial de Saúde (OMS) como um tumor cístico benigno, advindo do epitélio odontogênico e do ectomesênquima, podendo estar associado ao desenvolvimento da lâmina dentária, sendo incluído pela mesma entidade no grupo de cistos odontogênicos e não-odontogênicos do desenvolvimento (ARRUDA; ABREU, 2018; WRIGHT; VERED, 2017).

Trata-se de uma lesão rara que acomete maxila e mandíbula com leve predomínio do gênero masculino na faixa dos 30 anos (ARRUDA et al., 2018; CHRCANOVIC; GOMEZ, 2016). Geralmente, a lesão é intraóssea e expansível, o que propicia o aumento de volume facial nos contornos de tecidos moles sobrejacente à estrutura óssea acometida, de forma assintomática. Radiograficamente, o COC se apresenta como uma lesão radiolúcida com um halo radiopaco unilocular ou multilocular em região anterior de ambos maxilares (LI; YU, 2003), podendo provocar, nos dentes adjacentes à lesão, deslocamento das raízes dentárias e/ou reabsorções radiculares (CHINDASOMBATJAROEN; POOMSAWAT; KLONGNOI, 2012).

Irani; Foroughi (2017) analisaram 52 casos de COC (31 masculinos e 21 femininos) e os resultados indicaram que, aproximadamente, $31 \%$ e $8 \%$ estavam associados a odontoma e ameloblastoma, respectivamente. Dessa forma, o COC pode se manifestar de forma isolada ou associada a outros processos patológicos odontogênicos (odontoma,

\footnotetext{
* Faculdade de Odontologia, Hospital e Maternidade Therezinha de Jesus, Faculdade de Ciências Médicas e da Saúde de Juiz de Fora, FCMS/JF - SUPREMA, Juiz de Fora, MG.

**Área de Cirurgia e Traumatologia Bucomaxilofacial, Faculdade de Odontologia de Piracicaba, Universidade Estadual de Campinas, FOP - UNICAMP, Piracicaba, SP. E-mail: ctbmf.marliere@gmail.com

*** Serviço de Cirurgia e Traumatologia Bucomaxilofacial, Hospital Universitário Pedro Ernesto, Universidade do Estado do Rio de Janeiro, HUPE - UERJ, Rio de Janeiro, RJ.
} 
ameloblastoma e ceratocisto odontogênico), o que evidencia uma condição com certa diversidade e complexidade no processo patogênico (SANTOS et al., 2018).

Histopatologicamente, o COC corresponde a uma lesão incomum de desenvolvimento que se origina do epitélio odontogênico, caracterizada por uma camada basal bem definida no revestimento epitelial, apresentando camada sobrejacente com presença de células retículo estreladas e presença de células fantasmas no interior do revestimento epitelial ou cápsula fibrosa, podendo ocorrer nas formas intraóssea e extraóssea com a mesma probabilidade (LEE; KIM, 2014; LI; YU, 2003).

O objetivo deste relato de caso foi descrever a configuração clínica e histopatológica do cisto odontogêncio calcificante, bem como apresentar o tratamento cirúrgico e acompanhamento clínico e radiográfico pós-operatório.

\section{Relato de Caso}

Paciente do gênero feminino, 11 anos de idade, compareceu ao Serviço de Cirurgia e Traumatologia Bucomaxilofacial da Universidade do Estado do Rio de Janeiro, queixando-se de aumento de volume em região paranasal, lado esquerdo, sem sintomatologia dolorosa. $\mathrm{Na}$ anamnese, a responsável pela paciente não relatou nenhuma alteração sistêmica ou fatores de comorbidades, sendo solicitados exames laboratoriais pré-operatórios (hemograma, coagulograma e glicemia em jejum). Os resultados dos exames não indicaram qualquer anormalidade.

A responsável pela paciente assinou o termo de ciência e consentimento informado para realização do procedimento cirúrgico e autorizou a possível publicação para fins científicos.

\subsection{Exame clínico e radiográfico}

No exame clínico extraoral, observou-se a presença de aumento de volume no lado esquerdo da maxila, provocando deslocamento da base alar do nariz e o desaparecimento do sulco nasolabial (Figura 1A). $\mathrm{Na}$ avaliação intraoral, notou-se a extensão da lesão, que se encontrava entre a distal do elemento 22 até a distal do elemento 24 , sem alteração em superfície de mucosa alveolar e gengival, além de indeterminação quanto ao tempo de evolução (Figura 1B).

Para complementação dos exames clínicos, foram realizadas radiografia panorâmica e oclusal de maxila, as quais evidenciaram lesão radiolúcida unilocular circundada por um halo radiopaco, com aproximadamente 2,5 centímetros em diâmetro, deslocamento de raízes dentárias, sem aparente envolvimento de seio maxilar e abaulamento de parede anterior de maxila à esquerda (Figuras 2 e 3).

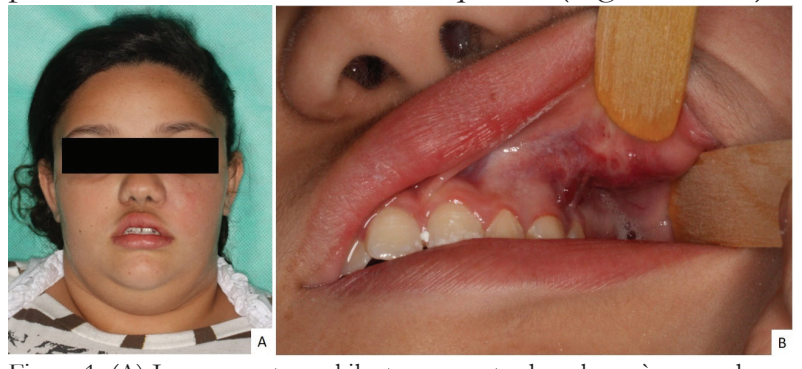

Figura 1: (A) Imagem extraoral ilustra aumento de volume à esquerda; (B) Imagem intraoral evidencia aumento volumétrico em fundo de vestíbulo.

Fonte: Os autores (2011).
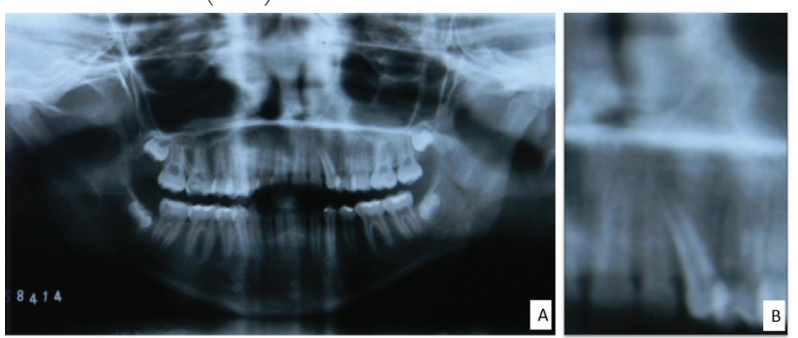

Figura 2: (A) Imagem da radiografia panorâmica pré-operatória. (B) Aumento da imagem radiográfica.

Fonte: Os autores (2011).

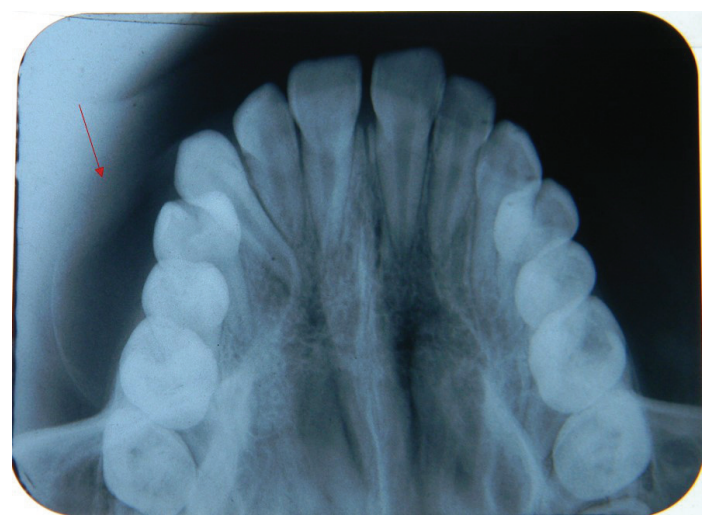

Figura 3: Imagem oclusal evidenciou abaulamento de parede maxilar (seta vermelha).

Fonte: Os autores (2011).

\subsection{Abordagem cirúrgica}

Inicialmente, a paciente realizou bochecho com clorexidina $0,12 \%$ e foi submetida a antissepsia de pele, com clorexidina $2 \%$ em região periférica de lábios e aposição dos campos cirúrgicos. Foi realizada incisão de aproximadamente $3 \mathrm{~cm}$ em mucosa gengival livre ( $5 \mathrm{~mm}$ acima da gengiva inserida) para descolamento do retalho mucoperiosteal, sob anestesia local com lidocaína 2\% com epinefrina 1:100000 (Nova DFL, Rio de Janeiro, Brasil).

Em seguida, foi identificada a região da lesão pelo abaulamento da parede anterior da maxila. Para exposição da lesão intra-óssea foi realizada ostectomia com motor elétrico tipo brushless (Driller, São Paulo, Brasil), peça multiplicadora (Kavo Dental, Santa Catarina, Brasil) e broca esférica número 06 (KG 
Sorensen, São Paulo, Brasil), com irrigação por soro fisiológico a 0,9\% (Eurofarma, Rio de Janeiro, Brasil) (Figura 4A). A lesão foi enucleada por dissecção, seguida de curetagem de sítio ósseo e finalização com ostectomia marginal de aproximadamente 1 a $2 \mathrm{~mm}$ da estrutura óssea adjacente à lesão, por meio de broca cirúrgica esférica e irrigação com soro fisiológico 0,9\% (Figura 4B e C). As peças anatomopatológicas apresentaram características macroscópicas compatíveis de uma lesão cística, contendo líquido no interior e revestida por cápsula fibrosa e firme, sendo armazenadas em formol a $10 \%$ e enviadas à análise histopatológica (figura 4D).

No pós-operatório imediato, a paciente foi medicada com amoxicilina $500 \mathrm{mg}$ via oral de $08 \mathrm{em}$ 08 horas, durante 07 dias, ibuprofeno $600 \mathrm{mg}$ via oral de 08 em 08 horas por 03 dias e solução de digluconato de clorexidina $0,12 \%$ para bochecho por 05 dias.
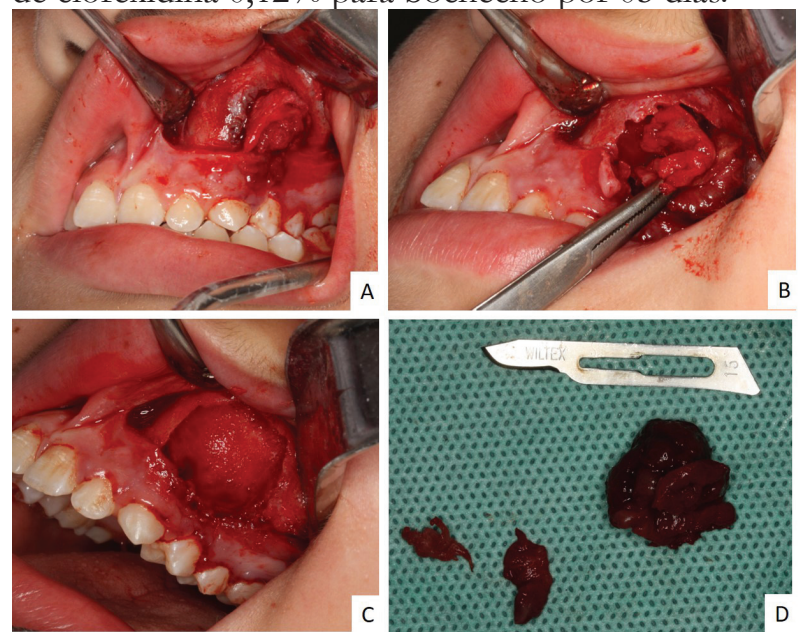

Figura 4: (A) Exposição da lesão intra-óssea. (B) Remoção da lesão pela enucleação por dissecção. (C) Ostectomia de 1 a 2 mm de margem óssea e sítio ósseo após completa remoção. (D) Lesão removida através de enucleação por dissecção.

Fonte: Os autores (2011)

\subsection{Análise histopatológica}

No exame histopatológico, a coloração de hematoxilina e eosina (H/E) foi utilizada para avaliação microscópica, sendo observado uma cápsula fibrosa com revestimento de epitélio odontogênico ameloblástico, apresentando células fantasmas e células basais de revestimento cuboides com sua camada sobrejacente de epitélio arranjada frouxamente (Figura 5 A, B e C). O laudo histopatológico confirmou o diagnóstico de COC.

Após confirmação do diagnóstico, realizou-se acompanhamento clínico por mais de 05 anos e não havia nenhuma evidência clínica de recorrência (Figura 6).

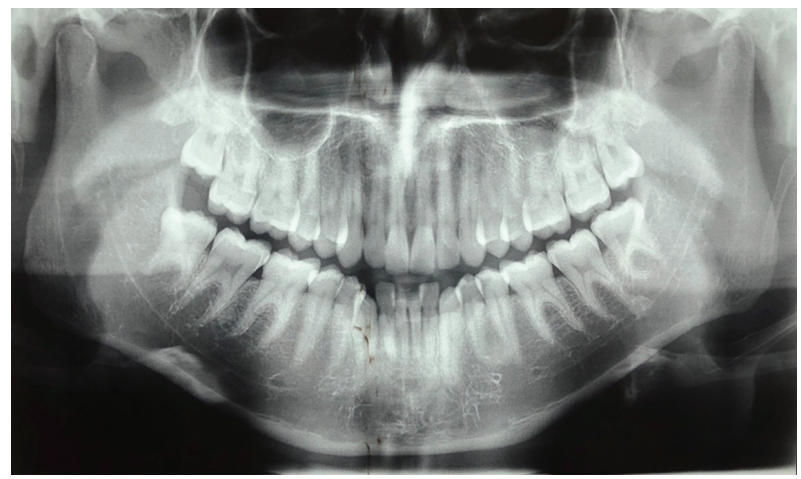

Figura 6: Radiografia panorâmica após 05 anos de pós-operatório Fonte: Os autores (2018)

\section{Discussão}

Desde o início dos anos 60, a caracterização do COC é complexa devido a sua diversidade de apresentação clínica, pois há possibilidade de associação a tumores de origem odontogênica (odontoma, ameloblastoma e tumor odontogênico adenomatóide). Além disso, histopatologicamente, apresenta características compatíveis a cistos e tumores benignos odontogênicos, sendo observado um processo de calcificação distrófico com formação de tecido dentinóide em sua cápsula fibrosa (GORLIN et al., 1962; TARAKJI et al., 2015; ZORNOSA; MULLER, 2010; SHAH; PATEL; PANDYA, 2018).

Há um fator predisponente para evolução de suas variantes císticas e neoplásicas, como o carcinoma odontogênico de células fantasmas (TARAKJI et al., 2015; ZORNOSA; MULLER, 2010). No presente relato de caso, os sinais radiográficos (radiopacidade unilocular com abaulamento de cortical circunscrita e bem localizada) associados ao padrão da execução
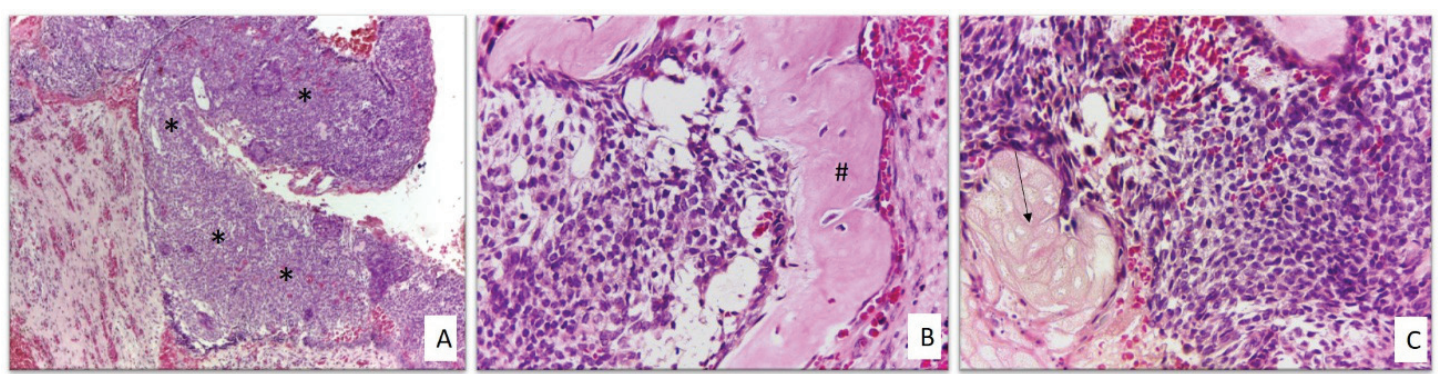

Figura 5: Características da análise histopatológica (A)*Revestimento epitelial odontogênico ameloblástico (H/E 200x); (B) \#Material dentinóide eosinofílico (H/E 400x); (C) Seta indicando grandes células fantasmas eosinofílicas (H/E 400x).

Fonte: Os autores (2011) 
cirúrgica revelaram que o quadro seria sugestivo de uma lesão de caráter mais cístico do que tumoral, sendo determinante a confirmação diagnóstica (excluindo associação com outras lesões) e acompanhamento clínico pós-operatório para excluir evolução neoplásica como citado anteriormente.

Independente de a classificação do COC ter sido heterogênea ao longo do tempo, o entendimento das características clínicas, radiográficas e histopatológicas são fundamentais para determinar a abordagem cirúrgica previsível e acompanhamento clínico após intervenção. Isso pode ser evidenciado por meio do estudo realizado por Silva et al. (2014), o qual afirma que compreender COC como uma lesão patológica única é controverso, devendo ser levado em consideração a sua diversidade de apresentação e a possibilidade de associação com outros tumores odontogênicos. O caso aqui relatado apresentava características clínicas similares a outras patologias, sendo essencial o diagnóstico histopatológico.

Santos et al. (2018) evidenciaram que aproximadamente $65 \%$ dos casos de COC localizamse na mandíbula em pacientes adultos, o que divergiu do caso relatado. Entretanto, a apresentação em maxila em pacientes jovens foi descrita por Reyes et al. (2007) e Kler; Palaska; Shetty (2009), evidenciando compatibilidade com a descrição do presente caso, pois houve compatibilidade em localização (região anterior de maxila) em pacientes na primeira e segunda década de vida.

Com relação ao exame clínico, deve-se levar em consideração a associação da lesão com as raízes dos dentes, podendo haver reabsorção radicular entre $75 \%$ a $77 \%$ dos casos de COC, sobretudo de incisivos e caninos erupcionados ou impactados (DANIELS, 2004). Chindasombatjaroen et al. (2012), ao analisarem, retrospectivamente, radiografias de pacientes diagnosticados com COC, observaram que $44 \%$ dos pacientes diagnosticados apresentaram deslocamento dentários, e 33\% reabsorção radicular.

No presente relato, ao considerar a avaliação radiográfica, foi observado deslocamento de raízes dos dentes 23 e 24 (adjacentes a lesão) sem sinais de reabsorção radicular, optando assim pelo tratamento cirúrgico descrito com preservação dos dentes. Nesse contexto, considerou-se importante a manutenção dos dentes adjacentes ao COC, pois promoveria a recuperação da paciente com preservação da oclusão, da estética e da função mastigatória, assim como propuseram Abu-Mostafa; Abassi (2017) quando envolvidos em casos de cistos dentígeros.

Independente da variação entre as características de localização, clínicas e de associação a outras condições patológicas, o tratamento do COC deve ser individualizado, mas o principal objetivo deve ser a remoção da lesão de forma a evitar recidivas (UTUMI et al., 2012). Nesse sentido, a enucleação por dissecção, associada à curetagem e ostectomia marginal, foi o tratamento cirúrgico para resolução da maioria dos casos intraósseos do COC (SOUZA et al., 2007), sendo o mesmo procedimento realizado no caso descrito neste estudo.

Segundo Stoelinga (2012), as biópsias incisionais prévias ao tratamento cirúrgico podem ser insuficientes para elucidação diagnóstica, pois as coletas de materiais podem ser comprometidas, gerando resultados histopatológicos inespecíficos. Além disso, sugerem que lesões de caráter cístico (uniloculares) nas regiões de maxila e mandíbula devam ser enucleadas completamente, quando não representam riscos a estrutura óssea e danos a tecidos neurossensoriais. Favoravelmente, o caso relatado demonstra que o tratamento cirúrgico permitiu a resolução segura em única etapa cirúrgica, sem necessidade de biópsia incisional prévia, o que foi justificável pela configuração clínica e radiográfica da lesão.

Outras terapias para resolução do caso, como a marsupialização e descompressão com enucleação secundária do cisto poderiam ter sido utilizadas para diminuir o tamanho ou defeito da lesão intraóssea (POGREL, 2003; STOELINGA, 2012). Entretanto, a utilização desses recursos terapêuticos apresentam como desvantagem períodos mais longos de tratamento por falhas nos artefatos que conduzem a descompressão ou crescimento tecidual na região marsupial (CHAPELLE; STOELINGA, BROUNS, 2004). Portanto, a dimensão do COC era unilocular e bem localizada, o que justificou a intenção terapêutica em um único estágio cirúrgico por enucleação e ostectomia marginal, sem prejudicar os tecidos ósseos e dentários adjacente.

Para a reparação no caso apresentado, foi realizada enucleação da lesão juntamente com ostectomia do tecido ósseo adjacente, com o objetivo de minimizar recidivas por resíduos patogênicos teciduais, tendo em vista que tais intervenções são apropriadas para a maioria dos casos (KIM; CHOI; KO, 2016). Ademais, foi realizado acompanhamento pós-operatório regular, como recomendado, para observação de possíveis recorrências, principalmente nos primeiros anos. A ausência de sinais e/ou sintomas de recidiva corroboraram com a adequada técnica de remoção da lesão (STOELINGA, 2001).

\section{Conclusão}

O relato de caso de COC apresentou perfil clínico que poderia ser confundido com outras lesões 
císticas e/ou tumorais de origem odontogênica ou de desenvolvimento, sendo essencial a elucidação por meio de análise histopatológica. A abordagem cirúrgica foi fundamental para diagnóstico e tratamento, excluindo associação com outras patologias. Após acompanhamento clínico e radiográfico, não houve recorrência do COC em região maxilar, corroborando com a adequação da técnica cirúrgica empregada no tratamento.

\section{Clinical and histopathological features of calcifying odontogenic cyst: case report}

\section{Abstract}

Calcifying odontogenic Cyst (COC) has been considering as benign pathological ill and rare occurrence in maxilla and mandible, there been showed odontogenic epithelial cystic lining demonstrating ghost cells with a propensity to calcify. In this article, we described the clinical and histopathological features from case reported who had undergone surgical approach and postoperative follow-up. A 11 year-old-girl had presented volumetric increased in left maxilla area near nasolabial fold. The radiography images had indicated radiolucent lesion, well-circumscribed and wide on cortical maxillary bone, which was undergone enucleation associated with marginal osteotomy on the bone cortical walls. The histopathological features showed cystic lining with odontogenic epithelium containing ghost cells and some areas with eosinophilic matrix material compatible dentinoid. Thus, it was evidenced a clinical features of COC which could be confused with other cystic or tumoral lesions, there being essential on diagnosis and treatment. After clinical and radiographic follow-up during 07 years, there was no recurrence of the COC.

Keywords: Calcifying Odontogenic Cyst. Odontogenesis. Oral Pathology. Oral Surgery.

\section{REFERENCIAS}

ABU-MOSTAFA, N.; ABASSI, A. Marsupialization of a large dentigerous cyst in the mandible with orthodontic extrusion of three impacted teeth. A case report. Journal of Clinical and Experimental Dentistry, v. 9, n. 5, p. 1162-1166, jul. 2017.

ARRUDA, J. A.; ABREU, L. G. A multicentre study of 268 cases of calcifying odontogenic cysts and a literature review. Oral Diseases, v. 24, n. 7, p. 1282-1293, mai. 2018.

ARRUDA, J. A. et al. Calcifying odontogenic cyst: A 26-year retrospective clinicopathological analysis and immunohistochemical study. Journal of Clinical and Experimental Dentistry, v. 10, n. 6, p. 542-547, abr. 2018.

CHAPELLE, K. A.; STOELINGA, P. J.; BROUNS, J. J. Rational approach to diagnosis and treatment of ameloblastomas and odontogenic keratocysts. The British Journal Oral Maxillofacial Surgery, v. 42, n. 5, p. 381-390, out. 2004.

CHINDASOMBATJAROEN, J.; POOMSAWAT, S.; KLONGNOI, B. Calcifying cystic odontogenic tumor associated with other lesions: case report with cone-beam computed tomography findings. Oral Surgery, Oral Medicine, Oral Pathology and Oral Radiology, v. 113, n.3, p. 414-420, mar.2012. CHINDASOMBATJAROEN, J. et al. Calcifying cystic odontogenic tumor and adenomatoid odontogenic tumor: radiographic evaluation. Oral Surgery, Oral Medicine, Oral Pathology and Oral Radiology, v. 114, n. 6, p. 796-803, dez. 2012.
CHRCANOVIC, B. R.; GOMEZ, R. S. Peripheral calcifying cystic odontogenic tumour and peripheral dentinogenic ghost cell tumour: an updated systematic review of 117 cases reported in the literature. Acta Odontologica Scandinavica, v. 74, n.8, p. 591-597, sep. 2016.

DANIELS, J. S. M. Recurrent calcifying odontogenic cyst involving the maxillary sinus. Oral Surgery, Oral Medicine, Oral Pathology, Oral Radiology and Endodontics, v. 98, n. 6, p. 660-664, abr. 2004.

GORLIN, R. J. et al. The calcifying ddontogenic cyst-A possible analogue of the cutaneous calcifying epithelioma of Malherbe an analaysis of fifteen cases. Oral Surgery, Oral Medicine and Oral Pathology, v. 15, n.10, p. 1235-1243, out. 1962.

IRANI, S.; FOROUGHI, F. Histologic variants of calcifying odontogenic cyst: a study of 52 cases. The Journal of Contemporary Dental Practice, v. 18, n. 8, p. 688-694, ago. 2017.

KIM, Y.; CHOI, B. E.; KO, S. O. Conservative approach to recurrent calcifying cystic odontogenic tumor occupying the maxillary sinus: a case report. Journal of the Korean Association of Oral and Maxillofacial Surgeons, v. 42, n. 5, p. 315-320, out. 2016.

KLER, S. et al. Intraosseous calcifying cystic odontogenic tumor. Journal of Oral and Maxillo Facial Pathology, v. 13, n. 1, p. 27-29, jan./jun. 2009.

HU Revista, Juiz de Fora, v. 43, n. 3, p. 415-420, out./dez. 2017 
LEE, S. K.; KIM, Y. S. Current concepts and occurrence of epithelial odontogenic tumours: II. Calcifying epithelial odontogenic tumour versus ghost cell odontogenic tumours derived from calcifying odontogenic cyst. The Korean Journal of Pathology, v. 48, n. 3, p. 175-187, jun. 2014.

LI, T. J.; YU, S. F. Clinicopathologic spectrum of the so-called calcifying odontogenic cysts: a study of 21 intraosseous cases with reconsideration of the terminology and classification. The American Journal of Surgical Pathology, v. 27, n. 3, p. 372384, mar. 2003.

POGREL, M. A. Decompression and marsupialization as a treatment for the odontogenic keratocyst. Oral and Maxillofacial Surgery Clinics of North America, v. 15, n. 3, p. 415-427, aug. 2003.

REYES, D. et al. Odontogenic calcificant cystic tumor: A report of two clinical cases. Medicina Oral Patología Oral y Cirurgía Bucal, v. 12, p. 126-129, nov. 2006.

SANTOS, H. B. P. et al. Calcifying odontogenic cyst with extensive areas of dentinoid: uncommon Case Report and update of main findings. Case Reports in Pathology, v. 2018, p. 1-4, may. 2018.

SHAH, U. et al. Ameloblastomatous calcifying ddontogenic cyst: a rare entity. Annals of Maxillofacial Surgery, v. 8, n. 1, p. 108115, jan./jun. 2018.

SILVA, Y. S. et al. Effectiveness of surgical decompression in the treatment of a calcifying cystic odontogenic tumor. Autopsy Case Reports, v. 4, n. 4, p. 43-49, mar. 2014.
SOUZA, L. N. et al. Conservative treatment of calcifying odontogenic cyst: report of 3 cases. Journal of Oral Maxillofacial Surgery, v. 65, n. 11, p. 2353-2356, nov. 2007.

STOELINGA, P. J. W. Long-term follow-up on keratocysts treated according to a defined protocol. International Journal of Oral and Maxillofacial Surgery, v. 30, n. 1, p. 14-25, fev. 2001.

STOELINGA, P. J. W. The management of aggressive cysts of the jaws. Journal of Maxillofacial and Oral Surgery, v. 11, n. 1, p. 2-12, mar. 2012.

TARAKJI, B.; ASHOK, N.; ALZOGHAIBI, I. et al. Malignant transformation of calcifying cystic odontogenic tumour - a review of literature. Contemporary Oncology, v. 19, n.3, p. 184186, jul. 2015.

UTUMI, E. R. et al. Different manifestations of calcifying cystic odontogenic tumor. Einstein, v. 10, n. 3, p. 366-370, set. 2012.

WRIGHT, J. M.; VERED, M. Update from the 4th edition of the world Health organization classification of head and neck tumours: odontogenic and maxillofacial bone tumors. Head and Neck Pathology, v. 11, n. 1, p. 68-77, mar. 2017.

ZORNOSA, X.; MÜLLER, S. Calcifying Cystic Odontogenic Tumor. Head and Neck Pathology, v. 4, n. 4, p. 292-294, dez. 2010. 